\title{
Rotor Spectra, Berry Phases, and Monopole Fields: from Graphene to Antiferromagnets and QCD
}

\author{
Uwe-Jens Wiese \\ Institute for Theoretical Physics, ETH Zürich \\ Schafmattstrasse 32, CH-8093 Zürich, Switzerland \\ Center for Research and Education in Fundamental Physics \\ Institute for Theoretical Physics, Bern University, Switzerland \\ E-mail: wiese@itp.unibe.ch
}

\begin{abstract}
Nonrelativistic electrons hopping on the honeycomb lattice of graphene emerge as massless Dirac fermions. When the on-site repulsion between electrons on a honeycomb lattice exceeds a critical value, as it is the case for the dehydrated precursor of the high-temperature superconductor $\mathrm{Na}_{2} \mathrm{CoO}_{2} \times y \mathrm{H}_{2} \mathrm{O}$, the system spontaneously breaks its $S U(2)_{s}$ spin symmetry and becomes an antiferromagnet. The emergence of antiferromagnetism is analogous to the spontaneous breakdown of the $S U(2)_{L} \times S U(2)_{R}$ chiral symmetry in QCD. Just as the low-energy physics of pions and nucleons is described by baryon chiral perturbation theory, magnons and holes in an antiferromagnet are also described by a systematic low-energy effective theory. Both the chiral condensate in QCD and the staggered magnetization in an antiferromagnet act as a quantum mechanical rotor when the theory is put in a finite volume. When a nucleon is propagating through the QCD vacuum or when a hole is doped into an antiferromagnet, a Berry phase arises from a geometric monopole gauge field and the angular momentum of the rotor is quantized in half-integer units. The finite-size effects of the rotor spectrum depend on the low-energy parameters of the corresponding effective theories.
\end{abstract}

The XXVI International Symposium on Lattice Field Theory

July 14 - 19, 2008

Williamsburg, Virginia, USA 


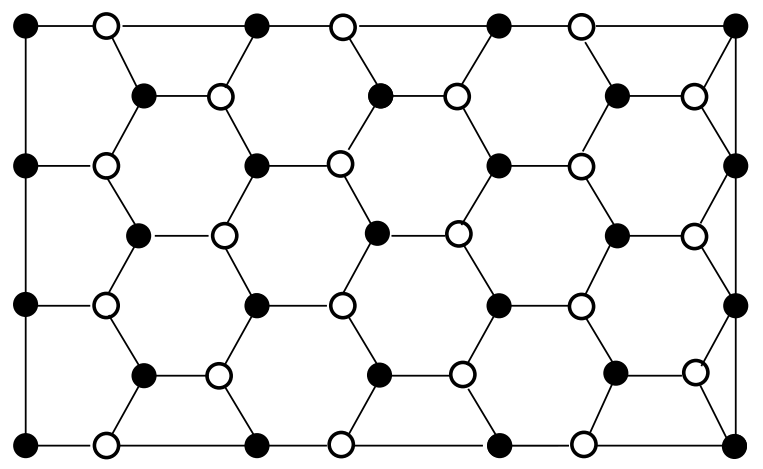

Figure 1: Bipartite non-Bravais honeycomb lattice consisting of two triangular Bravais sublattices.

\section{From Graphene to $\mathrm{Na}_{2} \mathrm{CoO}_{2} \times y \mathrm{H}_{2} \mathrm{O}$}

The dynamics of electrons hopping on a lattice is strongly influenced by the lattice geometry. Two interesting condensed matter systems on a honeycomb lattice are graphene - a 2dimensional sheet of graphite - and the dehydrated precursor of the high-temperature superconductor $\mathrm{Na}_{2} \mathrm{CoO}_{2} \times y \mathrm{H}_{2} \mathrm{O}$.

\subsection{Free Fermions on the Honeycomb Lattice}

The honeycomb lattice, illustrated in figure 1, is a bipartite non-Bravais lattice which consists of two triangular Bravais sublattices A and $\mathrm{B}$. The basis vectors that generate the triangular sublattices are given by

$$
a^{1}=\sqrt{3} a\left(\frac{\sqrt{3}}{2}, \frac{1}{2}\right), a^{2}=\sqrt{3} a(0,1),
$$

where $a$ is the lattice spacing. The hexagonal Brillouin zone $B Z$ of the honeycomb lattice is illustrated in figure 2. The honeycomb lattice has a number of discrete symmetries. Translations by the vectors $a^{i}$ are denoted by $D_{i}$. Counter-clockwise rotations by 60 degrees around the center of a hexagon are denoted by $O$, and reflections at the $x$-axis going through the center of rotation are denoted by $R$. Translations by other distance vectors, rotations by other angles or around other centers, and reflections with respect to other axes can be obtained as combinations of the elementary symmetry operations $D_{1}, D_{2}, O$, and $R$.

Free fermions hopping between nearest-neighbor sites $\langle x y\rangle$ on the honeycomb lattice are described by the Hamiltonian

$$
H_{t}=-t \sum_{\langle x y\rangle}\left(c_{x}^{\dagger} c_{y}+c_{y}^{\dagger} c_{x}\right), \quad c_{x}^{\dagger}=\left(c_{x \uparrow}^{\dagger}, c_{x \downarrow}^{\dagger}\right), \quad c_{x}=\left(\begin{array}{c}
c_{x \uparrow} \\
c_{x \downarrow}
\end{array}\right) .
$$

The creation and annihilation operators $c_{x s}^{\dagger}$ and $c_{x s}$ for fermions with spin $s=\uparrow, \downarrow$ at a lattice site $x$ obey standard anti-commutation relations. Obviously, the Hamiltonian commutes with the unitary transformations that generate the discrete symmetries $D_{i}, O$, and $R$ of the honeycomb lattice. In addition, an $S U(2)_{s}$ symmetry is generated by the total spin

$$
\vec{S}=\sum_{x} \vec{S}_{x}=\sum_{x} c_{x}^{\dagger} \frac{\vec{\sigma}}{2} c_{x}
$$




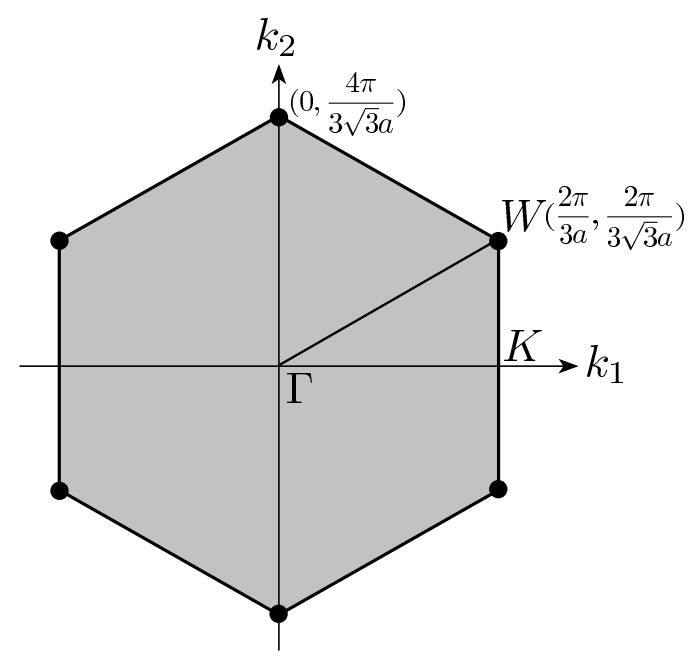

Figure 2: The momentum space of a honeycomb lattice, which is a doubly-covered Brillouin zone dual to the two triangular sublattices $A$ and $B$.

where $\vec{\sigma}$ are the Pauli matrices. The spin symmetry plays the role of an internal symmetry, like quark flavor in QCD. The $U(1)_{Q}$ symmetry corresponding to fermion number (relative to halffilling) is generated by the charge operator

$$
Q=\sum_{x} Q_{x}=\sum_{x}\left(c_{x}^{\dagger} c_{x}-1\right)
$$

It is straightforward to diagonalize the above Hamiltonian by going to momentum space using

$$
c^{A}(k)=\frac{1}{\sqrt{A_{B Z}}} \sum_{x \in A} c_{x} \exp (-i k x), \quad c^{B}(k)=\frac{1}{\sqrt{A_{B Z}}} \sum_{x \in B} c_{x} \exp (-i k x) .
$$

Here $A_{B Z}=8 \pi^{2} / 3 \sqrt{3} a^{2}$ is the area of the Brillouin zone. In momentum space the Hamiltonian takes the form

$$
\begin{aligned}
H_{t} & =\sum_{s=\uparrow, \downarrow} \int_{B Z} d^{2} k\left(c_{s}^{A \dagger}(k), c_{s}^{B^{\dagger}}(k)\right)\left(\begin{array}{cc}
0 & H_{A B}(k) \\
H_{A B}(k)^{*} & 0
\end{array}\right)\left(\begin{array}{c}
c_{s}^{A}(k) \\
c_{s}^{B}(k)
\end{array}\right), \\
H_{A B}(k) & =-t\left[\exp \left(-i k_{1} a\right)+\exp \left(i\left(k_{1}+\sqrt{3} k_{2}\right) \frac{a}{2}\right)+\exp \left(i\left(k_{1}-\sqrt{3} k_{2}\right) \frac{a}{2}\right)\right] .
\end{aligned}
$$

Finally, diagonalizing the $2 \times 2$ matrix yields the energy eigenvalues

$$
E_{s, \pm}(k)= \pm\left|H_{A B}(k)\right|, \quad s=\uparrow, \downarrow .
$$

The energy of single-particle states vanishes, i.e. $E_{s, \pm}\left(k^{f}\right)=0$ with $f=\alpha, \beta$, for

$$
k^{\alpha}=\left(0, \frac{4 \pi}{3 \sqrt{3} a}\right), k^{\beta}=\left(0,-\frac{4 \pi}{3 \sqrt{3} a}\right) .
$$

The points $k^{\alpha}$ and $k^{\beta}$ are located at the corners of the hexagonal Brillouin zone illustrated in figure 2. The other four corners of the Brillouin zone are periodic copies of $k^{\alpha}$ and $k^{\beta}$. These points 
form the centers of two Dirac cones of massless fermions. Altogether, there are four "flavors" of two-component Dirac fermions with $f=\alpha, \beta$ and $s=\uparrow, \downarrow$. Expanding the Hamiltonian near the points $k^{\alpha}$ and $k^{\beta}$ one obtains two single-particle Dirac Hamiltonians

$$
H_{t}\left(k^{\alpha}+p\right)=-\left(p_{1} \sigma_{2}+p_{2} \sigma_{1}\right) c_{F}+\mathscr{O}\left(p^{2}\right), \quad H_{t}\left(k^{\beta}+p\right)=-\left(p_{1} \sigma_{2}-p_{2} \sigma_{1}\right) c_{F}+\mathscr{O}\left(p^{2}\right),
$$

with the Fermi-velocity $c_{F}=\frac{3}{2} t a$.

\subsection{The $t$ - $J$ Model on the Honeycomb Lattice}

When one endows the free fermions of the previous subsection with an on-site Coulomb repulsion that exceeds a critical value, the $S U(2)_{s}$ spin symmetry breaks down spontaneously to its $U(1)_{s}$ subgroup by the formation of a non-zero staggered magnetization. This is realized in the dehydrated antiferromagnetic precursor of the high-temperature superconductor $\mathrm{Na}_{2} \mathrm{CoO}_{2} \times y \mathrm{H}_{2} \mathrm{O}$. At large Coulomb repulsion, doubly-occupied sites cost a large amount of energy, and the physics is captured by the $t-J$ model with the Hamiltonian

$$
H_{t J}=P\left[-t \sum_{\langle x y\rangle}\left(c_{x}^{\dagger} c_{y}+c_{y}^{\dagger} c_{x}\right)+J \sum_{\langle x y\rangle} \vec{S}_{x} \cdot \vec{S}_{y}\right] P .
$$

Here $P$ is a projection operator that eliminates doubly occupied sites from the fermionic Hilbert space, and $J>0$ is the antiferromagnetic exchange coupling. The $t$ - $J$ model is still invariant under the symmetries $S U(2)_{s}, D_{i}, O$, and $R$.

At half-filling, i.e. with one fermion per lattice site, hopping is completely Pauli-blocked and the $t$ - $J$ model reduces to the quantum Heisenberg model with the Hamiltonian

$$
H_{J}=J \sum_{\langle x y\rangle} \vec{S}_{x} \cdot \vec{S}_{y}
$$

As we will see in the next section, cluster algorithm simulations of the Heisenberg model on the honeycomb lattice show that it has an antiferromagnetic ground state in which the $S U(2)_{s}$ spin symmetry is spontaneously broken down to $U(1)_{s}$. The corresponding Goldstone bosons are two massless spinwaves, also known as magnons. As summarized in table 1, there are many analogies between antiferromagnets and QCD. In particular, magnons are a condensed matter analog of pions, and electrons or holes doped into an antiferromagnet are analogous to nucleons or anti-nucleons in QCD. In fact, just as nucleons are massive due to spontaneous chiral symmetry breaking, the previously massless Dirac fermions become massive in the antiferromagnetic phase due to spontaneous $S U(2)_{s}$ symmetry breaking. Indeed, the single-hole dispersion relation of the $t$ - $J$ model on the honeycomb lattice, illustrated in figure 3 , corresponds to massive fermions.

\section{Low-Energy Effective Theories for Antiferromagnets}

Just as the low-energy physics of QCD is described by chiral perturbation theory, antiferromagnets are described by an analogous low-energy effective field theory. In particular, the pure magnon effective theory is analogous to pion chiral perturbation theory, while the effective theory for magnons coupled to doped holes is analogous to baryon chiral perturbation theory. 


\begin{tabular}{|c|c|c|}
\hline & QCD & Antiferromagnetism \\
\hline \hline broken phase & hadronic vacuum & antiferromagnetic phase \\
\hline global symmetry & chiral symmetry & spin rotations \\
\hline symmetry group $G$ & $S U(2)_{L} \otimes S U(2)_{R}$ & $S U(2)_{s}$ \\
\hline unbroken subgroup $H$ & $S U(2)_{L=R}$ & $U(1)_{s}$ \\
\hline Goldstone boson & pion & magnon \\
\hline Goldstone field in $G / H$ & $U(x) \in S U(2)$ & $\vec{e}(x) \in S^{2}$ \\
\hline order parameter & chiral condensate $\langle\bar{\psi} \psi\rangle$ & staggered magnetization $\mathscr{M}_{s}$ \\
\hline coupling strength & pion decay constant $F_{\pi}$ & spin stiffness $\rho_{s}$ \\
\hline propagation speed & velocity of light & spin-wave velocity $c$ \\
\hline conserved charge & baryon number $U(1)_{B}$ & electric charge $U(1)_{Q}$ \\
\hline charged particle & nucleon or anti-nucleon & electron or hole \\
\hline long-range force & pion exchange & magnon exchange \\
\hline dense phase & nuclear or quark matter & high- $T_{C}$ superconductor \\
\hline microscopic description & lattice QCD & $t$-J model \\
\hline $\begin{array}{c}\text { effective description } \\
\text { of Goldstone bosons }\end{array}$ & chiral perturbation & magnon effective \\
\hline $\begin{array}{c}\text { effective description } \\
\text { of charged fields }\end{array}$ & baryon chiral & theory \\
\hline
\end{tabular}

Table 1: Analogies between QCD and antiferromagnetism.

\subsection{Low-Energy Effective Theory for Magnons}

Due to the spontaneous breaking of the $S U(2)_{s}$ spin symmetry down to its $U(1)_{s}$ subgroup, the low-energy physics of antiferromagnets is governed by two massless magnons. The description of the low-energy magnon physics was pioneered by Chakravarty, Halperin, and Nelson in [1]. In analogy to chiral perturbation theory for the pseudo-Goldstone pions in QCD, a systematic low-energy effective field theory for magnons was developed in $[2,3,4,5]$. The staggered magnetization of an antiferromagnet is described by a unit-vector field $\vec{e}(x)$ in the coset space $S U(2)_{s} / U(1)_{s}=S^{2}$, i.e.

$$
\vec{e}(x)=(\sin \theta(x) \cos \varphi(x), \sin \theta(x) \sin \varphi(x), \cos \theta(x)) .
$$

Here $x=\left(x_{1}, x_{2}, t\right)$ denotes a point in $(2+1)$-dimensional space-time. To leading order, the Euclidean magnon low-energy effective action takes the form

$$
S[\vec{e}]=\int d^{2} x d t \frac{\rho_{s}}{2}\left(\partial_{i} \vec{e} \cdot \partial_{i} \vec{e}+\frac{1}{c^{2}} \partial_{t} \vec{e} \cdot \partial_{t} \vec{e}\right),
$$

where the index $i \in\{1,2\}$ labels the two spatial directions and $t$ refers to the Euclidean timedirection. The parameter $\rho_{s}$ is the spin stiffness and $c$ is the spin wave velocity.

In the $\varepsilon$-regime of magnon chiral perturbation theory, the finite-temperature and finite-volume effects of the staggered susceptibility have been worked out by Hasenfratz and Niedermayer at the 


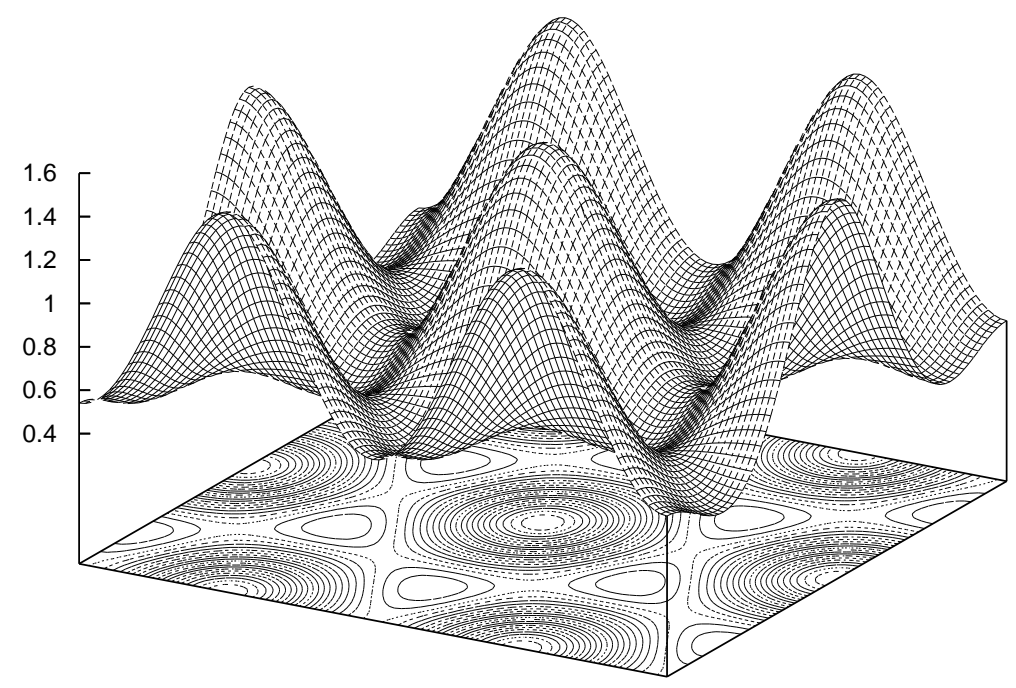

Figure 3: The energy-momentum dispersion relation $E(k)$ for a single hole in the $t$-J model on the honeycomb lattice.

two-loop level [6] and are given by

$$
\chi_{s}=\frac{\mathscr{M}_{s}^{2} L^{2} \beta}{3}\left\{1+2 \frac{c}{\rho_{s} L l} \beta_{1}(l)+\left(\frac{c}{\rho_{s} L l}\right)^{2}\left[\beta_{1}(l)^{2}+3 \beta_{2}(l)\right]+\mathscr{O}\left(\frac{1}{L^{3}}\right)\right\} .
$$

Here $\mathscr{M}_{s}$ is the staggered magnetization density. The uniform susceptibility takes the form

$$
\chi_{u}=\frac{2 \rho_{s}}{3 c^{2}}\left\{1+\frac{1}{3} \frac{c}{\rho_{s} L l} \widetilde{\beta}_{1}(l)+\frac{1}{3}\left(\frac{c}{\rho_{s} L l}\right)^{2}\left[\widetilde{\beta}_{2}(l)-\frac{1}{3} \widetilde{\beta}_{1}(l)^{2}-6 \psi(l)\right]+\mathscr{O}\left(\frac{1}{L^{3}}\right)\right\}
$$

Here $l=(\beta c / L)^{1 / 3}$ determines the shape of a space-time box of size $L \times L \times \beta$, with $\beta c \approx L$. The functions $\beta_{i}(l), \widetilde{\beta}_{i}(l)$, and $\psi(l)$ are known shape coefficients [6].

In the very low temperature limit, one enters the cylindrical regime of space-time volumes with $\beta c \gg L$ in which the $\delta$-expansion of chiral perturbation theory applies. The cylindrical spacetime geometry is illustrated in figure 4 . In this case, the staggered magnetization acts as a quantum mechanical rotor governed by the Lagrange function [6]

$$
\mathscr{L}=\frac{\Theta}{2} \partial_{t} \vec{e} \cdot \partial_{t} \vec{e}=\frac{\Theta}{2}\left[\left(\partial_{t} \theta\right)^{2}+\sin ^{2} \theta\left(\partial_{t} \varphi\right)^{2}\right]
$$

Integrating out the fast non-zero modes of the staggered magnetization at one loop, the moment of inertia results as

$$
\Theta=\frac{\rho_{s} L^{2}}{c^{2}}\left[1+\frac{3.900265}{4 \pi}\left(\frac{c}{\rho_{s} L}\right)+\mathscr{O}\left(\frac{1}{L^{2}}\right)\right] .
$$

The momenta conjugate to $\theta$ and $\varphi$ are

$$
p_{\theta}=\frac{\delta \mathscr{L}}{\delta \partial_{t} \theta}=\Theta \partial_{t} \theta, \quad p_{\varphi}=\frac{\delta \mathscr{L}}{\delta \partial_{t} \varphi}=\Theta \sin ^{2} \theta \partial_{t} \varphi
$$




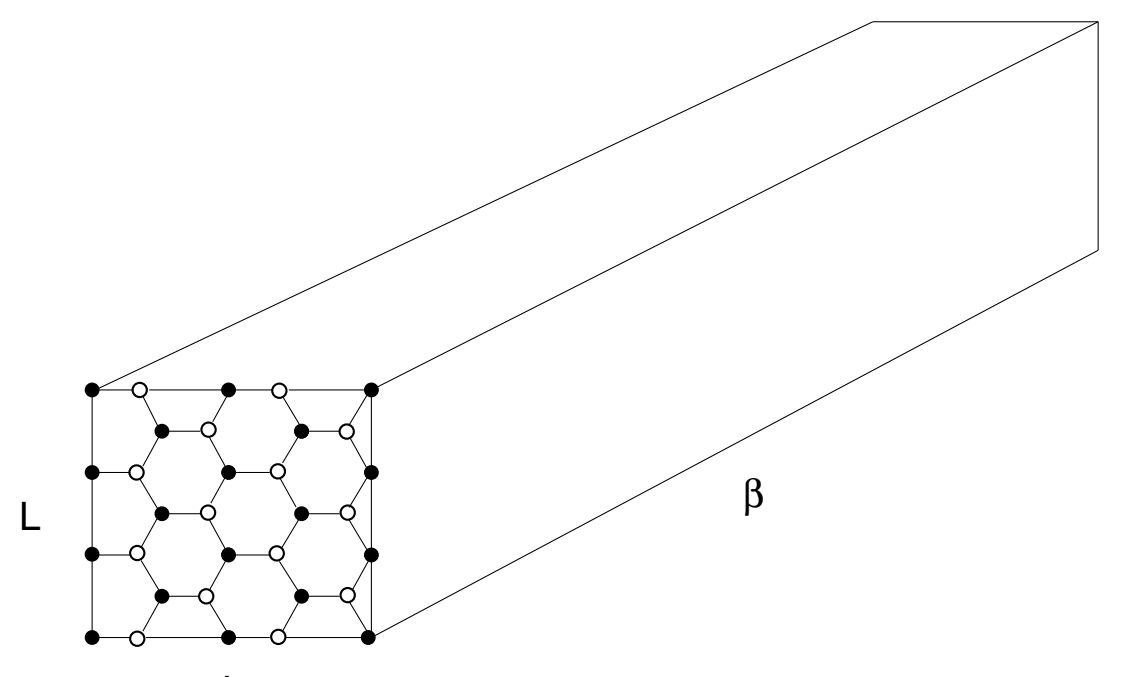

Figure 4: Cylindrical space-time geometry with temporal extent $\beta c \gg L$.

and the Hamiltonian as well as its energy spectrum result as

$$
H=-\frac{1}{2 \Theta}\left(\frac{1}{\sin \theta} \partial_{\theta}\left[\sin \theta \partial_{\theta}\right]+\frac{1}{\sin ^{2} \theta} \partial_{\varphi}^{2}\right)=\frac{\vec{L}^{2}}{2 \Theta}, \quad E_{l}=\frac{l(l+1)}{2 \Theta}, \quad l \in\{0,1,2, \ldots\} .
$$

The probability distribution of the uniform magnetization $M^{3}$ is then given by

$$
p\left(M^{3}\right)=\frac{1}{Z} \sum_{l \geq\left|M^{3}\right|} \exp \left(-\beta E_{l}\right), \quad Z=\sum_{l=0}^{\infty}(2 l+1) \exp \left(-\beta E_{l}\right) .
$$

\subsection{Determination of the Low-Energy Parameters of the Undoped System}

In order to determine the low-energy parameters $\mathscr{M}_{s}, \rho_{s}$, and $c$, numerical simulations of the Heisenberg model on the honeycomb lattice have been performed in [7], both in cubical and in cylindrical space-time geometries, using a very efficient loop-cluster algorithm $[8,9,10]$. A simultaneous fit of the Monte Carlo data for the susceptibilities $\chi_{s}$ and $\chi_{u}$ to eq.(2.3) and eq.(2.4) is shown in figure 5 . The fitted values of the low-energy parameters are

$$
\widetilde{\mathscr{M}_{s}}=\frac{3 \sqrt{3}}{4} \mathscr{M}_{s} a^{2}=0.2688(3), \quad \rho_{s}=0.102(2) J, \quad c=1.297(16) J a
$$

For comparison, on the square lattice the corresponding staggered magnetization per spin is $\widetilde{\mathscr{M}_{s}}=$ 0.3074(4) $[9,10]$. Due to the smaller coordination number of the honeycomb lattice, quantum fluctuations (which reduce the order parameter $\widetilde{\mathscr{M}_{s}}$ ) are larger than on the square lattice.

Once the values of the low-energy parameters $\widetilde{\mathscr{M}}_{s}, \rho_{s}$, and $c$ have been fixed in the cubical regime, the effective theory can be tested in the cylindrical regime. Figure 6 compares the effective theory result for the probability distribution $p\left(M^{3}\right)$ of eq.(2.9) with Monte Carlo data [7]. The observed excellent agreement - which does not rely on any additional adjustable parameters confirms the quantitative correctness of the effective theory. Numerical determinations of lowenergy parameters have also been successful in $O(N)$ models with classical spin variables [11]. 

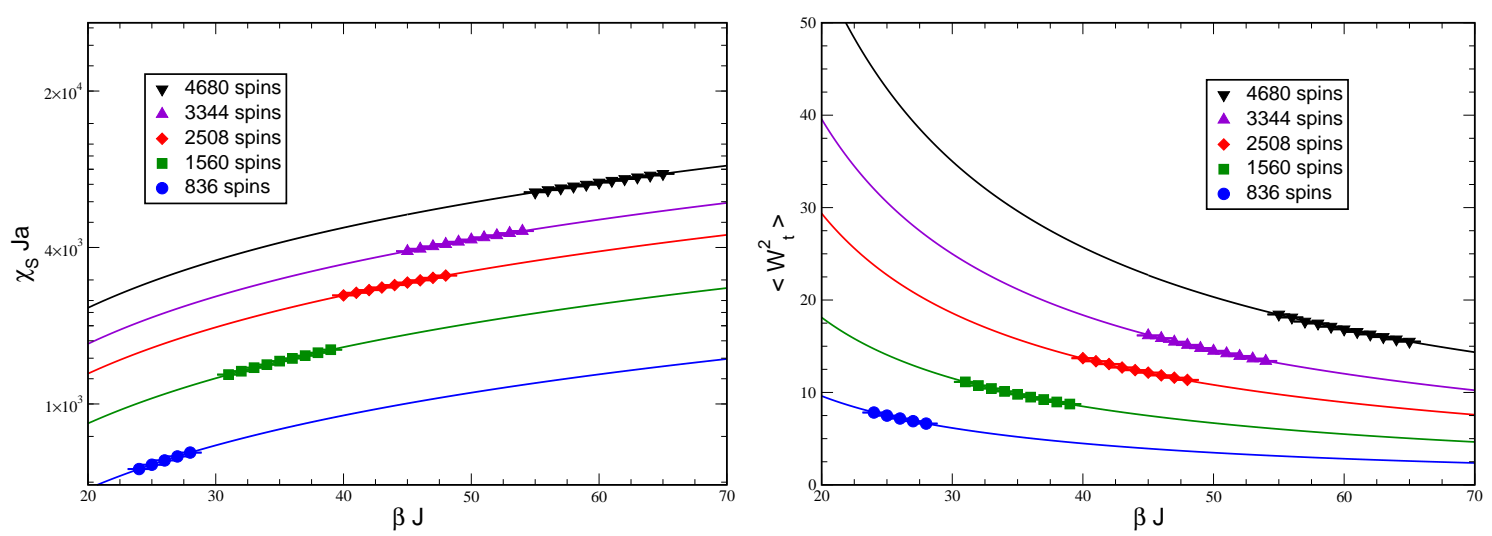

Figure 5: Fit of the finite-size and finite-temperature effects of the staggered and uniform susceptibilities $\chi_{\text {s }}$ and $\chi_{u}$ to analytic results of the effective theory in the cubical regime.

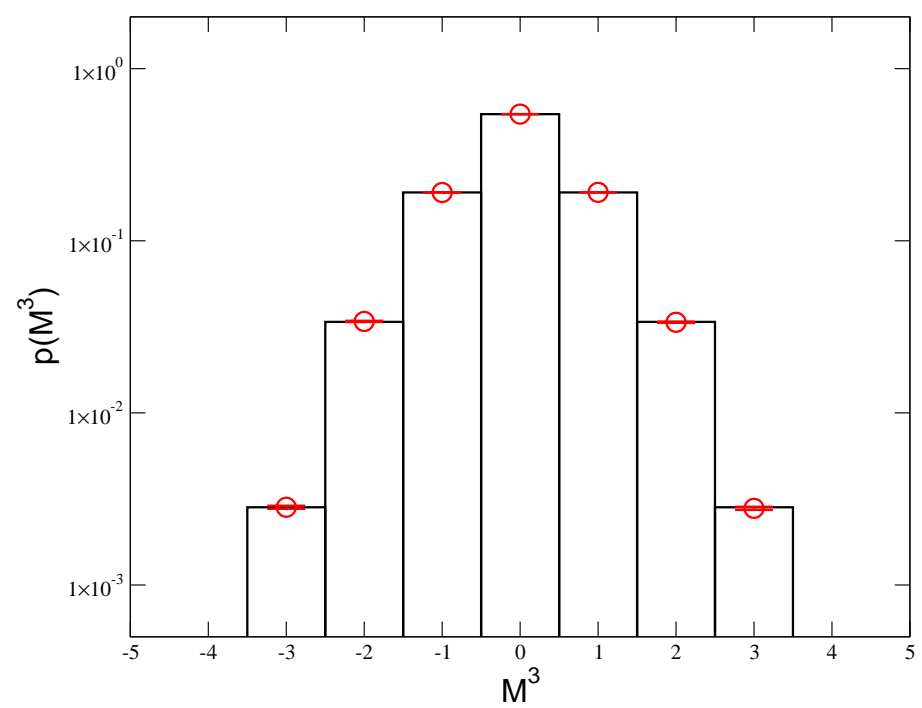

Figure 6: Comparison of the effective theory prediction for the probability distribution $p\left(M^{3}\right)$ of eq.(2.9) with Monte Carlo data for the Heisenberg model on a honeycomb lattice with 836 spins at $\beta J=60$. The open circles are the Monte Carlo data while the histogram is the effective theory prediction.

\subsection{Effective Field Theory for Holes and Magnons}

The description of holes doped into an antiferromagnet on the square lattice was pioneered by Shraiman and Siggia [12]. Motivated by the success of baryon chiral perturbation theory for QCD, a systematic low-energy effective field theory for magnons and holes in an antiferromagnet on the square lattice was constructed in $[13,14]$. This theory has been used in a detailed analysis of twohole states bound by one-magnon exchange $[14,15]$ as well as of spiral phases in the staggered magnetization [16], which are a condensed matter analog of pion condensation in dense nuclear matter. The systematic effective field theory investigations have also been extended to electron- 
doped antiferromagnets [17]. Similarly, an effective theory for holes doped into an antiferromagnet on the honeycomb lattice has been constructed in [18].

Magnons are coupled to holes through a nonlinear realization of the spontaneously broken $S U(2)_{s}$ symmetry [13]. First, one diagonalizes the magnon field by a unitary transformation $u(x) \in$ $S U(2)$, i.e.

$$
u(x) \vec{e}(x) \cdot \vec{\sigma} u(x)^{\dagger}=\sigma_{3}, \quad u_{11}(x) \geq 0 .
$$

Under a global $S U(2)_{s}$ transformation $g$, the diagonalizing field $u(x)$ transforms as

$$
u(x)^{\prime}=h(x) u(x) g^{\dagger}, \quad u_{11}(x)^{\prime} \geq 0
$$

which implicitly defines the nonlinear symmetry transformation

$$
h(x)=\exp \left(i \alpha(x) \sigma_{3}\right)=\left(\begin{array}{cc}
\exp (i \alpha(x)) & 0 \\
0 & \exp (-i \alpha(x))
\end{array}\right) \in U(1)_{s} .
$$

Introducing the traceless anti-Hermitean field

$$
v_{\mu}(x)=u(x) \partial_{\mu} u(x)^{\dagger}=i v_{\mu}^{a}(x) \sigma_{a}, \quad v_{\mu}^{ \pm}(x)=v_{\mu}^{1}(x) \mp i v_{\mu}^{2}(x),
$$

the field $v_{\mu}(x)$ decomposes into an Abelian "gauge" field $v_{\mu}^{3}(x)$ and two "charged" vector fields $v_{\mu}^{ \pm}(x)$. The Abelian "gauge" transformation acts on the composite vector fields as

$$
v_{\mu}^{3}(x)^{\prime}=v_{\mu}^{3}(x)-\partial_{\mu} \alpha(x), \quad v_{\mu}^{ \pm}(x)^{\prime}=v_{\mu}^{ \pm}(x) \exp ( \pm 2 i \alpha(x)) .
$$

As one sees in figure 3 , in the $t-J$ model on the honeycomb lattice holes occur in pockets centered at lattice momenta $k^{\alpha}=-k^{\beta}=\left(0, \frac{4 \pi}{3 \sqrt{3} a}\right)$, and their copies in the periodic Brillouin zone. In the effective theory, the holes are described by Grassmann-valued fields $\psi_{s}^{f}(x)$ carrying a "flavor" index $f=\alpha, \beta$ that characterizes the corresponding hole pocket. The index $s= \pm$ denotes spin parallel (+) or antiparallel ( - ) to the local staggered magnetization. Under the various symmetry operations the hole fields transform as

$$
\begin{aligned}
S U(2)_{s}: & \psi_{ \pm}^{f}(x)^{\prime}=\exp ( \pm i \alpha(x)) \psi_{ \pm}^{f}(x), \\
U(1)_{Q}: & { } \psi_{ \pm}^{f}(x)=\exp (i \omega) \psi_{ \pm}^{f}(x), \\
D_{i}: & { }^{D i} \psi_{ \pm}^{f}(x)=\exp \left(i k_{j}^{f} a_{j}^{i}\right) \psi_{ \pm}^{f}(x), \\
O: & { }^{o} \psi_{ \pm}^{\alpha}(x)=\mp \exp \left(\mp i \varphi(O x) \pm i \frac{2 \pi}{3}\right) \psi_{\mp}^{\beta}(O x), \\
& { }^{O} \psi_{ \pm}^{\beta}(x)=\mp \exp \left(\mp i \varphi(O x) \mp i \frac{2 \pi}{3}\right) \psi_{\mp}^{\alpha}(O x), \\
R: & { }^{R} \psi_{ \pm}^{\alpha}(x)=\psi_{ \pm}^{\beta}(R x), \quad{ }^{R} \psi_{ \pm}^{\beta}(x)=\psi_{ \pm}^{\alpha}(R x), \\
T: & { }^{T} \psi_{ \pm}^{\alpha}(x)=\exp (\mp i \varphi(T x)) \psi_{ \pm}^{\beta \dagger}(T x), \quad{ }^{T} \psi_{ \pm}^{\beta}(x)=\exp (\mp i \varphi(T x)) \psi_{ \pm}^{\alpha \dagger}(T x) .
\end{aligned}
$$

with

$$
\begin{aligned}
& O x=O\left(x_{1}, x_{2}, t\right)=\left(\frac{1}{2} x_{1}-\frac{\sqrt{3}}{2} x_{2}, \frac{\sqrt{3}}{2} x_{1}+\frac{1}{2} x_{2}, t\right), \\
& R x=R\left(x_{1}, x_{2}, t\right)=\left(x_{1},-x_{2}, t\right), \quad T x=T\left(x_{1}, x_{2}, t\right)=\left(x_{1}, x_{2},-t\right),
\end{aligned}
$$


where $T$ denotes time-reversal. Here $U(1)_{Q}$ is the fermion number symmetry of the holes. Interestingly, in the effective continuum theory the location of holes in lattice momentum space manifests itself as a "charge" $k_{j}^{f} a_{j}^{i}$ under the displacement symmetry $D_{i}$.

Following the principles of chiral perturbation theory, the low-energy effective action of magnons and holes is constructed as a derivative expansion. Since the holes are heavy nonrelativistic fermions, one time-derivative counts like two spatial derivatives. The leading terms in the Lagrangian are given by

$$
\mathscr{L}=\sum_{\substack{f=\alpha, \beta \\ s=+,-}}\left[M \psi_{s}^{f \dagger} \psi_{s}^{f}+\psi_{s}^{f \dagger} D_{t} \psi_{s}^{f}+\frac{1}{2 M^{\prime}} D_{i} \psi_{s}^{f \dagger} D_{i} \psi_{s}^{f}+\Lambda \psi_{s}^{f \dagger}\left(i s v_{1}^{s}+\sigma_{f} v_{2}^{s}\right) \psi_{-s}^{f}\right] .
$$

It should be noted that $v_{i}^{ \pm}(x)$ contains one spatial derivative, such that magnons and holes are indeed derivatively coupled. In eq.(2.18), $M$ is the rest mass and $M^{\prime}$ is the kinetic mass of a hole, while $\Lambda$ is the leading hole-one-magnon coupling. The $\operatorname{sign} \sigma_{f}$ is + for $f=\alpha$ and - for $f=\beta$. The covariant derivative in eq.(2.18) takes the form

$$
D_{\mu} \psi_{ \pm}^{f}(x)=\partial_{\mu} \psi_{ \pm}^{f}(x) \pm i v_{\mu}^{3}(x) \psi_{ \pm}^{f}(x)
$$

\section{Rotor Spectra in the Presence of Fermions}

Berry phases and monopole fields are familiar from adiabatic processes in quantum mechanics [19]. For example, the slow rotation of the nuclei in a diatomic molecule is influenced by a geometric vector potential generated by the fast motion of the electrons, whose Abelian and non-Abelian monopole content was worked out in [20]. Corresponding Berry phases and monopole fields have been identified for antiferromagnets and for QCD in [21].

\subsection{Rotor Spectrum of an Antiferromagnet in the Presence of a Single Hole}

When a single hole is doped into the antiferromagnet, the spin of the system changes by $1 / 2$ and thus the angular momentum of the resulting rotor must then be quantized in half-integer units. The half-integer quantization is a result of Berry phases and monopole fields. The leading terms in the low-energy Lagrange function of a hole with small momentum $\vec{p}$ are given by

$$
\mathscr{L}=\frac{\Theta}{2} \partial_{t} \vec{e} \cdot \partial_{t} \vec{e}+\psi^{f \dagger}\left[E(\vec{p})-i \partial_{t}+v_{t}^{3} \sigma_{3}+\lambda V_{t}\right] \psi^{f} .
$$

Here $\psi^{f}(t)$ is a two-component Grassmann-valued field describing fermions with spin parallel or anti-parallel to the local staggered magnetization. The fermion energy $E(\vec{p})$ as well as $\lambda$ can be determined by integrating out the non-zero momentum modes of the staggered magnetization. For hole-doped cuprates on the square as well as on the honeycomb lattice, the corresponding effective theories predict that $\lambda=0[14,18]$, while for other antiferromagnets in general $\lambda \neq 0[13]$. One obtains

$$
v_{t}^{3}=\sin ^{2} \frac{\theta}{2} \partial_{t} \varphi, \quad V_{t}=\frac{1}{2} \sin \theta\left(\cos \varphi \sigma_{1}+\sin \varphi \sigma_{2}\right) \partial_{t} \varphi+\frac{1}{2}\left(\sin \varphi \sigma_{1}-\cos \varphi \sigma_{2}\right) \partial_{t} \theta .
$$

These velocity-dependent terms give rise to a modification of the canonically conjugate momenta such that

$$
\Theta \partial_{t} \theta=p_{\theta}+i A_{\theta}, \quad \Theta \partial_{t} \varphi=\frac{1}{\sin ^{2} \theta}\left(p_{\varphi}+i A_{\varphi}\right),
$$




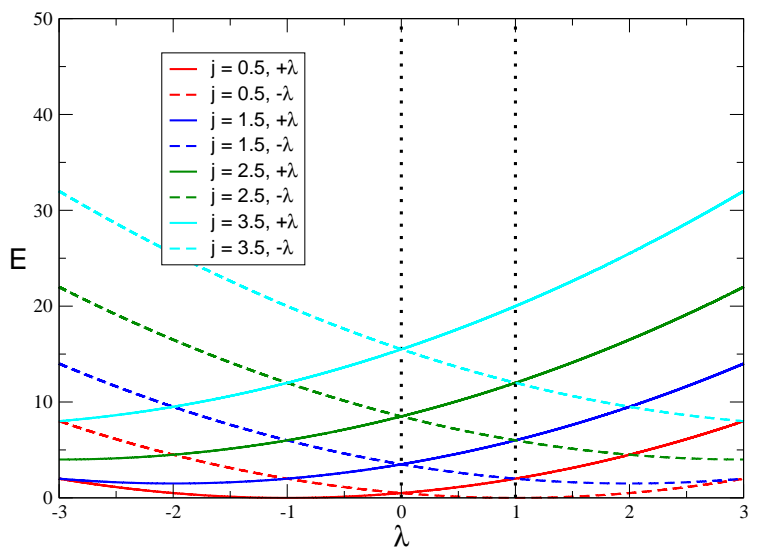

Figure 7: Rotor spectrum of an antiferromagnet in the presence of a single hole as a function of $\lambda$.

with the non-Abelian vector potential

$$
A_{\theta}=i \frac{\lambda}{2}\left(\sin \varphi \sigma_{1}-\cos \varphi \sigma_{2}\right), \quad A_{\varphi}=i \sin ^{2} \frac{\theta}{2} \sigma_{3}+i \frac{\lambda}{2} \sin \theta\left(\cos \varphi \sigma_{1}+\sin \varphi \sigma_{2}\right),
$$

and the corresponding field strength

$$
F_{\theta \varphi}=\partial_{\theta} A_{\varphi}-\partial_{\varphi} A_{\theta}+\left[A_{\theta}, A_{\varphi}\right]=i \frac{1-\lambda^{2}}{2} \sin \theta \sigma_{3} .
$$

Interestingly, the resulting geometric Berry gauge field is exactly the same as for a diatomic molecule [20]. For cuprates $(\lambda=0)$ the vector potential is Abelian and describes a monopole with quantized magnetic flux. For a general antiferromagnet (with $\lambda \neq 0$ ), on the other hand, the vector potential becomes non-Abelian and the flux is no longer quantized.

The resulting Hamilton operator then takes the form

$$
H(\lambda)=-\frac{1}{2 \Theta}\left\{\frac{1}{\sin \theta}\left(\partial_{\theta}-A_{\theta}\right)\left[\sin \theta\left(\partial_{\theta}-A_{\theta}\right)\right]+\frac{1}{\sin ^{2} \theta}\left(\partial_{\varphi}-A_{\varphi}\right)^{2}\right\}+E(\vec{p}) .
$$

The solution for the energy spectrum has been obtained in [21] along the lines of [20]. The Hamiltonian $H(0)$ (corresponding to $\lambda=0$ ) commutes with the angular momentum operators

$$
J_{ \pm}=\exp ( \pm i \varphi)\left( \pm \partial_{\theta}+i \cot \theta \partial_{\varphi}-\frac{1}{2} \tan \frac{\theta}{2} \sigma_{3}\right), \quad J_{3}=-i \partial_{\varphi}-\frac{\sigma_{3}}{2} .
$$

The Hamiltonian $H(0)$ together with its spectrum $E_{j}(0)$ is given by

$$
H(0)=\frac{1}{2 \Theta}\left(\vec{J}^{2}-\frac{1}{4}\right)+E(\vec{p}), \quad E_{j}(0)=\frac{1}{2 \Theta}\left[j(j+1)-\frac{1}{4}\right]+E(\vec{p}) .
$$

Here $j$ is a half-integer. In this case, each state is $2(2 j+1)$-fold degenerate because the fermion sectors + and - cost the same energy. The Hamiltonian with $\lambda \neq 0$ takes the form

$$
H(\lambda)=H(0)+\frac{1}{2 \Theta}\left(\lambda C+\frac{1}{2} \lambda^{2}\right)
$$


and still commutes with $\vec{J}$ of eq.(3.6). Here

$$
C=-i\left(\sin \varphi \partial_{\theta}+\frac{\cos \varphi}{\sin \theta} \partial_{\varphi}-\frac{1}{2} \sin \varphi \tan \frac{\theta}{2}\right) \sigma_{1}+i\left(\cos \varphi \partial_{\theta}-\frac{\sin \varphi}{\sin \theta} \partial_{\varphi}-\frac{1}{2} \cos \varphi \tan \frac{\theta}{2}\right) \sigma_{2},
$$

and $[C, \vec{J}]=0$. Using $C^{2}=\vec{J}^{2}+\frac{1}{4}$ one obtains the energy spectrum

$$
E_{j}(\lambda)=\frac{1}{2 \Theta}\left[j^{\prime}\left(j^{\prime}+1\right)+\frac{\lambda^{2}-1}{4}\right]+E(\vec{p}),
$$

with $j^{\prime}=j \pm \frac{\lambda}{2}$ and $j$ again being a half-integer. For $\lambda \neq 0$ the fermion sectors + and - get mixed and the previously degenerate $2(2 j+1)$ states are now split into two groups of $2 j+1$ degenerate states. Interestingly, for $\lambda= \pm 1$ the monopole field strength of eq.(3.4) vanishes and $E_{j}( \pm 1)=$ $\frac{1}{2 \Theta} j^{\prime}\left(j^{\prime}+1\right)$ with $j^{\prime}=j \pm \frac{1}{2}$. In that case, the rotor spectrum looks like the one of eq.(2.8) although the angular momentum $j$ is now a half-integer. The rotor spectrum as a function of $\lambda$ is illustrated in figure 7.

\subsection{Rotor Spectrum of QCD in the Single Nucleon Sector}

Let us now consider QCD with two massless flavors and thus with a spontaneously broken $S U(2)_{L} \times S U(2)_{R}$ chiral symmetry. When the theory is put in a finite spatial volume $L^{3}$, the chiral order parameter $U(t) \in S U(2)$ describes a quantum rotor with the moment of inertia given by $\Theta=F_{\pi}^{2} L^{3}$, where $F_{\pi}$ is the pion decay constant. The corresponding Hamiltonian is the Laplacian on the sphere $S^{3}$. The QCD rotor spectrum has been derived by Leutwyler [22] in the $\delta$-expansion of chiral perturbation theory as

$$
E_{l}=\frac{j_{L}\left(j_{L}+1\right)+j_{R}\left(j_{R}+1\right)}{\Theta}=\frac{l(l+2)}{2 \Theta} .
$$

In this case, $j_{L}=j_{R}$ with $l=j_{L}+j_{R} \in\{0,1,2, \ldots\}$ and each state is $\left(2 j_{L}+1\right)\left(2 j_{R}+1\right)=(l+1)^{2}$ fold degenerate. The low-energy dynamics of nucleons and pions is described by baryon chiral perturbation theory. When a nucleon with small momentum $\vec{p}=|\vec{p}| \vec{e}_{p}$ is propagating in the finite volume, the Lagrangian reads

$$
\mathscr{L}=\frac{\Theta}{4} \operatorname{Tr}\left[\partial_{t} U^{\dagger} \partial_{t} U\right]+\psi^{\dagger}\left[E(\vec{p})-i \partial_{t}-i v_{t}-i \lambda\left(\vec{\sigma} \cdot \vec{e}_{p}\right) a_{t}\right] \psi
$$

Here $\psi(t)$ is a Pauli spinor with a flavor index distinguishing protons and neutrons and $\frac{\vec{\sigma}}{2}$ is the nucleon spin. At tree level, $E(\vec{p})=M+\vec{p}^{2} / 2 M$ and $\lambda=g_{A}|\vec{p}| / M$, where $M$ is the mass and $g_{A}$ is the axial vector coupling of the nucleon. As for the antiferromagnet, the parameters $\Theta, E(\vec{p})$, and $\lambda$ get renormalized by the coupling to non-zero momentum pion modes. Here $u^{2}=U$ and

$$
v_{t}=\frac{1}{2}\left(u \partial_{t} u^{\dagger}+u^{\dagger} \partial_{t} u\right), \quad a_{t}=\frac{1}{2 i}\left(u \partial_{t} u^{\dagger}-u^{\dagger} \partial_{t} u\right) .
$$

Parameterizing

$$
\begin{aligned}
& U(t)=\cos \alpha(t)+i \sin \alpha(t) \vec{e}_{\alpha}(t) \cdot \vec{\tau}, \\
& \vec{e}_{\alpha}(t)=(\sin \theta(t) \cos \varphi(t), \sin \theta(t) \sin \varphi(t), \cos \theta(t)), \\
& \vec{e}_{\theta}(t)=(\cos \theta(t) \cos \varphi(t), \cos \theta(t) \sin \varphi(t),-\sin \theta(t)), \\
& \vec{e}_{\varphi}(t)=(-\sin \varphi(t), \cos \varphi(t), 0),
\end{aligned}
$$


and denoting the Pauli matrices for isospin by $\vec{\tau}$, one obtains

$$
v_{t}=i \sin ^{2} \frac{\alpha}{2}\left(\partial_{t} \theta \vec{e}_{\varphi}-\sin \theta \partial_{t} \varphi \vec{e}_{\theta}\right) \cdot \vec{\tau}, \quad a_{t}=\left(\frac{\partial_{t} \alpha}{2} \vec{e}_{\alpha}+\sin \alpha \frac{\partial_{t} \theta}{2} \vec{e}_{\theta}+\sin \alpha \sin \theta \frac{\partial_{t} \varphi}{2} \vec{e}_{\varphi}\right) \cdot \vec{\tau} .
$$

The resulting Hamilton operator takes the form

$$
\begin{aligned}
H(\lambda) & =-\frac{1}{2 \Theta}\left\{\frac{1}{\sin ^{2} \alpha}\left(\partial_{\alpha}-A_{\alpha}\right)\left[\sin ^{2} \alpha\left(\partial_{\alpha}-A_{\alpha}\right)\right]\right. \\
& \left.+\frac{1}{\sin ^{2} \alpha \sin \theta}\left(\partial_{\theta}-A_{\theta}\right)\left[\sin \theta\left(\partial_{\theta}-A_{\theta}\right)\right]+\frac{1}{\sin ^{2} \alpha \sin ^{2} \theta}\left(\partial_{\varphi}-A_{\varphi}\right)^{2}\right\}+E(\vec{p}),
\end{aligned}
$$

with the non-Abelian vector potential

$$
\begin{aligned}
& A_{\alpha}=i \frac{\lambda}{2}\left(\vec{\sigma} \cdot \vec{e}_{p}\right) \vec{e}_{\alpha} \cdot \vec{\tau}, \quad A_{\theta}=i\left(\sin ^{2} \frac{\alpha}{2} \vec{e}_{\varphi}+\frac{\lambda}{2}\left(\vec{\sigma} \cdot \vec{e}_{p}\right) \sin \alpha \vec{e}_{\theta}\right) \cdot \vec{\tau}, \\
& A_{\varphi}=i\left(-\sin ^{2} \frac{\alpha}{2} \sin \theta \vec{e}_{\theta}+\frac{\lambda}{2}\left(\vec{\sigma} \cdot \vec{e}_{p}\right) \sin \alpha \sin \theta \vec{e}_{\varphi}\right) \cdot \vec{\tau},
\end{aligned}
$$

and the corresponding field strength representing a non-Abelian monopole

$$
F_{\alpha \theta}=i \frac{1-\lambda^{2}}{2} \sin \alpha \vec{e}_{\varphi} \cdot \vec{\tau}, \quad F_{\theta \varphi}=i \frac{1-\lambda^{2}}{2} \sin ^{2} \alpha \sin \theta \vec{e}_{\alpha} \cdot \vec{\tau}, \quad F_{\varphi \alpha}=i \frac{1-\lambda^{2}}{2} \sin \alpha \sin \theta \vec{e}_{\theta} \cdot \vec{\tau} .
$$

The generators of $S U(2)_{L} \otimes S U(2)_{R}$ are given by

$$
\begin{aligned}
\vec{J}_{L} & =\frac{1}{2}(\vec{J}-\vec{K}), \quad \vec{J}_{R}=\frac{1}{2}(\vec{J}+\vec{K}), \quad J_{ \pm}=\exp ( \pm i \varphi)\left( \pm \partial_{\theta}+i \cot \theta \partial_{\varphi}\right)+\frac{\tau_{ \pm}}{2}, \\
K_{ \pm} & =\exp ( \pm i \varphi)\left(i \sin \theta \partial_{\alpha}+i \cot \alpha \cos \theta \partial_{\theta} \mp \frac{\cot \alpha}{\sin \theta} \partial_{\varphi} \mp \frac{i}{2} \tan \frac{\alpha}{2} \vec{e}_{\theta} \cdot \vec{\tau}+\frac{1}{2} \tan \frac{\alpha}{2} \cos \theta \vec{e}_{\varphi} \cdot \vec{\tau}\right), \\
J_{3} & =-i \partial_{\varphi}+\frac{\tau_{3}}{2}, \quad K_{3}=i\left(\cos \theta \partial_{\alpha}-\cot \alpha \sin \theta \partial_{\theta}\right)-\frac{1}{2} \tan \frac{\alpha}{2} \sin \theta \vec{e}_{\varphi} \cdot \vec{\tau} .
\end{aligned}
$$

The Hamiltonian $H(0)$ (with $\lambda=0$ ) and its spectrum $E_{j}(0)$ take the form

$$
H(0)=\frac{1}{2 \Theta}\left(\vec{J}^{2}+\vec{K}^{2}-\frac{3}{4}\right)+E(\vec{p}), \quad E_{j}(0)=\frac{1}{2 \Theta}\left[j(j+2)-\frac{1}{2}\right]+E(\vec{p}) .
$$

In this case, $j_{L}=j_{R} \pm \frac{1}{2}$ and $j=j_{L}+j_{R} \in\left\{\frac{1}{2}, \frac{3}{2}, \ldots\right\}$. Each state is $2\left(j+\frac{1}{2}\right)\left(j+\frac{3}{2}\right)$-fold degenerate because the states with spin up and spin down cost the same energy. The Hamiltonian with $\lambda \neq 0$ can be written as

$$
H(\lambda)=H(0)+\frac{1}{2 \Theta}\left(\lambda C+\frac{3}{4} \lambda^{2}\right),
$$

and it still commutes with $\vec{J}$ and $\vec{K}$. Here

$$
C=i\left(\vec{\sigma} \cdot \vec{e}_{p}\right)\left(\vec{e}_{\alpha} \partial_{\alpha}+\frac{1}{\sin \theta} \vec{e}_{\theta} \partial_{\theta}+\frac{1}{\sin \alpha \sin \theta} \vec{e}_{\varphi} \partial_{\varphi}-\tan \frac{\alpha}{2} \vec{e}_{\alpha}\right) \cdot \vec{\tau}
$$

and $[C, \vec{J}]=[C, \vec{K}]=0$. Using $C^{2}=\vec{J}^{2}+\vec{K}^{2}+\frac{3}{4}$ one finally obtains the energy spectrum

$$
E_{j}(\lambda)=\frac{1}{2 \Theta}\left[j^{\prime}\left(j^{\prime}+2\right)+\frac{\lambda^{2}-1}{2}\right]+E(\vec{p})
$$


with $j^{\prime}=j \pm \frac{\lambda}{2}$, where \pm refers to the spin eigenstates of $\vec{\sigma} \cdot \vec{e}_{p}$ with eigenvalues \pm 1 . Thus we see that for $\lambda \neq 0$ the degeneracy is partly lifted and there are now two groups of $\left(j+\frac{1}{2}\right)\left(j+\frac{3}{2}\right)$-fold degenerate states. Remarkably, for $\lambda= \pm 1$ the non-Abelian field strength of eq.(3.18) vanishes and $E_{j}( \pm 1)=\frac{1}{2 \Theta} j^{\prime}\left(j^{\prime}+2\right)$ with $j^{\prime}=j \pm \frac{1}{2}$. Just as for an antiferromagnet with $\lambda= \pm 1$, the QCD rotor spectrum then looks like the one of eq.(3.11) although the system now has fermion number one.

The study in the $\delta$-regime [21] complements other investigations of finite volume effects in the one-nucleon sector of QCD in the $p$ - [23, 24], $\varepsilon$ - [25], and $\varepsilon^{\prime}$-regimes [26] of chiral perturbation theory. A comparison of numerical lattice QCD data in the vacuum sector with finite volume predictions in the $\varepsilon$ - and $\delta$-regimes of chiral perturbation theory leads to an accurate determination of low-energy parameters such as $F_{\pi}[27,28]$. Before one could do the same in the single-nucleon sector, e.g. in order to determine $g_{A}$, one must match the volume-dependent parameters $\Theta, E(\vec{p})$, and $\lambda$ of the effective quantum mechanics to those of the infinite volume effective theory.

\section{Conclusions}

Antiferromagnets and QCD share a number of common features. In particular, systematic effective field theories capture the low-energy physics in both cases. The condensed matter analog of pions and nucleons in QCD are magnons and holes in doped antiferromagnets. Thus, the analog of baryon chiral perturbation theory is a systematic effective field theory for magnons and holes. The effective theories make quantitative predictions for finite-size and finite-temperature effects which can be tested in great detail in numerical simulations. While the $\varepsilon$-regime of chiral perturbation theory is more easily accessible in numerical simulations, the rotor spectra in the $\delta$-regime have intriguing theoretical features. In particular, in the sectors with a single hole doped into an antiferromagnet or a single nucleon propagating through the QCD vacuum, Berry gauge fields with Abelian and non-Abelian monopoles arise. In contrast to QCD, very efficient cluster algorithms are available for quantum antiferromagnets. In this way, the effective theory has been verified in great detail even at the 2-loop level. It is to be expected that a similar accuracy will eventually be reached in simulations of lattice QCD.

\section{Acknowledgments}

This talk is based on work done in collaboration with B. Bessire, S. Chandrasekharan, C. P. Hofmann, F. J. Jiang, F. Kämpfer, M. Nyfeler, M. Pepe, and M. Wirz, whom I like to thank for numerous interesting discussions and a very pleasant collaboration. The work was supported in part by funds provided by the Schweizerischer Nationalfonds. The "Center for Research and Education in Fundamental Physics" at Bern University is supported by the "Innovations- und Kooperationsprojekt C-13" of the Schweizerische Universitätskonferenz (SUK/CRUS). 


\section{References}

[1] S. Chakravarty, B. I. Halperin, and D. R. Nelson, Phys. Rev. B39 (1989) 2344.

[2] H. Neuberger and T. Ziman, Phys. Rev. B39 (1989) 2608.

[3] D. S. Fisher, Phys. Rev. B39 (1989) 11783.

[4] P. Hasenfratz and H. Leutwyler, Nucl. Phys. B343 (1990) 241.

[5] P. Hasenfratz and F. Niedermayer, Phys. Lett. B268 (1991) 231.

[6] P. Hasenfratz and F. Niedermayer, Z. Phys. B92 (1993) 91.

[7] F. J. Jiang, F. Kämpfer, M. Nyfeler, and U.-J. Wiese, arXiv:0807.2977 [cond-mat.str-el].

[8] H. G. Evertz, G. Lana, and M. Marcu, Phys. Rev. Lett. 70 (1993) 875.

[9] U.-J. Wiese and H.-P. Ying, Z. Phys. B93 (1994) 147.

[10] B. B. Beard and U.-J. Wiese, Phys. Rev. Lett. 77 (1996) 5130.

[11] A. Hasenfratz, K. Jansen, J. Jersák, C. B. Lang, H. Leutwyler, and T. Neuhaus, Z. Phys. C46 (1990) 257.

[12] B. I. Shraiman and E. D. Siggia, Phys. Rev. Lett. 60 (1988) 740; Phys. Rev. Lett. 61 (1988) 467; Phys. Rev. Lett. 62 (1989) 1564; Phys. Rev. B46 (1992) 8305.

[13] F. Kämpfer, M. Moser, and U.-J. Wiese, Nucl. Phys. B729 (2005) 317.

[14] C. Brügger, F. Kämpfer, M. Moser, M. Pepe, and U.-J. Wiese, Phys. Rev. B74 (2006) 224432.

[15] C. Brügger, F. Kämpfer, M. Pepe, and U.-J. Wiese, Eur. Phys. J. B53 (2006) 433.

[16] C. Brügger, C. P. Hofmann, F. Kämpfer, M. Pepe and U.-J. Wiese, Phys. Rev. B75 (2007) 014421.

[17] C. Brügger, C. P. Hofmann, F. Kämpfer, M. Moser, M. Pepe, and U.-J. Wiese, Phys. Rev. B75 (2007) 214405.

[18] F. J. Jiang, F. Kämpfer, C. P. Hofmann, and U.-J. Wiese, arXiv:0809.4998 [cond-mat.str-el].

[19] M. Berry, Proc. Roy. Soc. London, Ser. A392 (1984) 45.

[20] J. Moody, A. Shapere, and F. Wilczek, Phys. Rev. Lett. 56 (1986) 893.

[21] S. Chandrasekharan, F. J. Jiang, M. Pepe, and U.-J. Wiese, arXiv:cond-mat/0612252, to appear in Phys. Rev. B.

[22] H. Leutwyler, Phys. Lett. B189 (1987) 197.

[23] A. Ali-Khan et al., Nucl. Phys. B689 (2004) 175.

[24] G. Colangelo, A. Fuhrer, and C. Haefeli, Nucl. Phys. Proc. Suppl. 153 (2006) 41.

[25] P. F. Bedaque, H. W. Griesshammer, and G. Rupak, Phys. Rev. D71 (2005) 054015.

[26] W. Detmold and M. Savage, Phys. Lett. B599 (2004) 32.

[27] P. Hasenfratz, D. Hierl, V. Maillart, F. Niedermayer, A. Schäfer, C. Weiermann, and M. Weingart, PoS (LATTICE 2007) 077.

[28] JLQCD collaboration, H. Fukaya et al., Phys. Rev. D76 (2007) 054503, Phys. Rev. Lett. 98 (2007) 172001, Phys. Rev. D77 (2008) 074503. 